\title{
ON THE STRUCTURE OF SPLIT INVOLUTIVE HOM-LIE COLOR ALGEBRAS
}

\author{
VALIOLLAH KHALILI
}

\begin{abstract}
In this paper we study the structure of arbitrary split involutive regular Hom-Lie color algebras. By developing techniques of connections of roots for this kind of algebras, we show that such a split involutive regular Hom-Lie color algebra $\mathcal{L}$ is of the form $\mathcal{L}=\mathcal{U} \oplus \sum_{[\alpha] \in \Pi / \sim} I_{[\alpha]}$, with $\mathcal{U}$ a subspace of the involutive abelian subalgebra $\mathcal{H}$ and any $I_{[\alpha]}$, a well-described involutive ideal of $\mathcal{L}$, satisfying $\left[I_{[\alpha]}, I_{[\beta]}\right]=0$ if $[\alpha] \neq[\beta]$. Under certain conditions, in the case of $\mathcal{L}$ being of maximal length, the simplicity of the algebra is characterized and it is shown that $\mathcal{L}$ is the direct sum of the family of its minimal involutive ideals, each one being a simple split involutive regular Hom-Lie color algebra. Finally, an example will be provided to characterise the inner structure of split involutive Hom-Lie color algebras.
\end{abstract}

\section{INTRODUCTION}

The notion of Lie color algebras was introduced as generalized Lie algebras in 1960 by Ree in [11]. In 1979, Scheunert investigated the Lie color algebras from a purely mathematical point of view (see [14]). So far, many results for this kind of algebras have been considered in the frameworks of enveloping algebras cohomology, representations, and related problems (see [5, 10, 12, 13, 15, 16, 19]).

In 2012, Yuan [20] introduced the notion of a Hom-Lie color algebra which can be considered as an extension of Hom-Lie superalgebras to $\Lambda$-graded algebras, where $\Lambda$ is any additive abelian group. The pioneering works in these subjects are [1, 2, 17].

As is well-known, the class of the split algebras is especially related to addition quantum numbers, graded contractions and deformations. For instance, for a physical system, which displays a symmetry of Lie algebra $\mathcal{L}$, it is interesting to know in detail the structure of the split decomposition, because its roots can be seen as certain eigenvalues which are the additive quantum numbers characterizing the state of such a system. Determining the structure of split algebras will become more and more meaningful in the area of research in mathematical physics. Recently, in [6, 9, 8, 3, 21, the structure of arbitrary split Lie algebras, arbitrary

2010 Mathematics Subject Classification. 17B40, 17B55, $17 \mathrm{~B} 75$.

Key words and phrases. Lie color algebras; Hom-Lie color algebras; Root systems; Root spaces; Structure theory. 
split involutive Lie algebras, arbitrary split Lie color algebras, arbitrary split regular Hom-Lie algebras, and arbitrary split involutive regular Hom-Lie algebras have been determined by the techniques of connection of roots.

Our goal in this work is to study the structure of arbitrary split involutive regular Hom-Lie color algebras by the techniques of connection of roots. The results of this article are based on some works in [3, 7, 21.

Throughout this paper, split involutive regular Hom-Lie color algebras $\mathcal{L}$ are considered of arbitrary dimension and over an arbitrary base field $\mathbb{F}$, with characteristic zero. We also consider an additive abelian group $\Lambda$ with identity zero.

To close this introduction, we briefly outline the contents of the paper. In Section 2, we begin by recalling the necessary background on split involutive regular Hom-Lie color algebras. Section 3 develops techniques of connections of roots for split involutive regular Hom-Lie color algebras. We also show that such an arbitrary split involutive regular Hom-Lie color algebra $\mathcal{L}$ with a root system $\Pi$ is of the form $\mathcal{L}=\mathcal{U} \oplus \sum_{[\alpha] \in \Pi / \sim} I_{[\alpha]}$, with $\mathcal{U}$ a subspace of the involutive abelian subalgebra $\mathcal{H}$ and any $I_{[\alpha]}$, a well-described involutive ideal of $\mathcal{L}$, satisfying $\left[I_{[\alpha]}, I_{[\beta]}\right]=0$ if $[\alpha] \neq[\beta]$. In Section 4, we show that under certain conditions, in the case of $\mathcal{L}$ being of maximal length, the simplicity of the algebra is characterized and it is shown that $\mathcal{L}$ is the direct sum of the family of its minimal involutive ideals, each one being a simple split involutive regular Hom-Lie color algebra. Finally, Section 5 provides a concrete example which characterizes the inner structure of split involutive Hom-Lie color algebras.

\section{Preliminaries}

Let us begin with some definitions concerning graded algebraic structures. For a detailed discussion of this subject, we refer the reader to the literature [14]. Let $\Lambda$ be any additive abelian group. A vector space $V$ is said to be $\Lambda$-graded if there is a family $\left\{V_{\lambda}\right\}_{\lambda \in \Lambda}$ of vector subspaces such that $V=\bigoplus_{\lambda \in \Lambda} V_{\lambda}$. An element $v \in V$ is said to be homogeneous of degree $\lambda$ if $v \in V_{\lambda}, \lambda \in \Lambda$, and in this case, $\lambda$ is called the color of $v$. As usual, we denote by $|v|$ the color of an element $v \in V$. Thus, each homogeneous element $v$ in $V$ determines a unique group element $|v| \in \Lambda$ by $v \in V_{|v|}$. Fortunately, we can almost always drop the symbol "| |", since confusion rarely occurs.

Let $V=\bigoplus_{\lambda \in \Lambda} V_{\lambda}$ and $W=\bigoplus_{\lambda \in \Lambda} W_{\lambda}$ be two $\Lambda$-graded vector spaces. A linear mapping $f: V \longrightarrow W$ is said to be homogeneous of degree $\theta \in \Lambda$ if

$$
f\left(V_{\lambda}\right) \subset W_{\lambda+\theta}, \quad \forall \lambda \in \Lambda
$$

If in addition $f$ is homogeneous of degree zero, namely, $f\left(V_{\lambda}\right) \subset W_{\lambda}$ holds for any $\lambda \in \Lambda$, then we call $f$ even.

An algebra $A$ is said to be $\Lambda$-graded if its underlying vector space is $\Lambda$-graded, i.e., $A=\bigoplus_{\lambda \in \Lambda} A_{\lambda}$, and if $A_{\lambda} A_{\mu} \subset A_{\lambda+\mu}$, for $\lambda, \mu \in \Lambda$. A subalgebra of $A$ is said to be graded if it is graded as a subspace of $A$. 
Let $B$ be another $\Lambda$-graded algebra. A homomorphism $\varphi: A \longrightarrow B$ of $\Lambda$-graded algebras is a homomorphism of the algebra $A$ into the algebra $B$, which is an even mapping.

Definition 2.1 ([14]). Let $\Lambda$ be an abelian group. A map $\epsilon: \Lambda \times \Lambda \longrightarrow \mathbb{K} \backslash\{0\}$ is called a skew-symmetric bi-character on $\Lambda$ if for all $\lambda, \mu, \eta \in \Lambda$,

(i) $\epsilon(\lambda, \mu) \epsilon(\mu, \lambda)=1$

(ii) $\epsilon(\lambda+\mu, \eta)=\epsilon(\lambda, \eta) \epsilon(\mu, \eta)$,

(iii) $\epsilon(\lambda, \mu+\eta)=\epsilon(\lambda, \mu) \epsilon(\lambda, \eta)$.

The definition above implies that in particular, the following relations hold:

$$
\epsilon(\lambda, 0)=1=\epsilon(0, \lambda), \quad \epsilon(\lambda, \lambda)=1(\text { or }-1), \quad \forall \lambda \in \Lambda .
$$

Throughout this paper, if $x$ and $y$ are homogeneous elements of a $\Lambda$-graded vector space and $|x|$ and $|y|$, which are in $\Lambda$, denote respectively their degrees, then for convenience we write $\epsilon(x, y)$ instead of $\epsilon(|x|,|y|)$. It is worth mentioning that, unless otherwise stated, in the sequel all the graded spaces are over the same abelian group $\Lambda$ and the bi-character is the same for all structures.

Definition 2.2 ([20]). A Hom-Lie color algebra is a tuple $(\mathcal{L},[.,],. \phi, \epsilon)$ consisting of a $\Lambda$-graded vector space $\mathcal{L}$, an even bilinear map [.,.] : $\mathcal{L} \times \mathcal{L} \longrightarrow \mathcal{L}$ (i.e., $\left.\left[\mathcal{L}_{\lambda}, \mathcal{L}_{\mu}\right] \subset \mathcal{L}_{\lambda+\mu}\right)$, an even homomorphism $\phi: \mathcal{L} \longrightarrow \mathcal{L}$, and a bicharacter $\epsilon$ such that for homogeneous elements $x, y, z \in \mathcal{L}$ these conditions are satisfied:

(i) $[x, y]=-\epsilon(x, y)[y, x] \quad(\epsilon$-skew-symmetry),

(ii) $\sum_{\circlearrowleft_{x, y}, z} \epsilon(z, x)[\phi(x),[y, z]]=0 \quad(\epsilon$-Hom-Jacobi identity),

where $\circlearrowleft_{x, y, z}$ denotes summation over the cyclic permutation on $x, y, z$.

In particular, if $\phi$ is a morphism of Lie algebras (i.e., $\phi \circ[.,]=.[.,.] \circ \phi^{\otimes 2}$ ), then we call $(\mathcal{L},[.,],. \phi, \epsilon)$ a multiplicative Hom-Lie color algebra. A Hom-Lie color algebra is called regular if $\phi$ is an automorphism. We recover a color Lie algebra when we have $\phi=i d$; color Lie algebra is a generalization of Lie algebra and Lie superalgebra (if $\Lambda=\{0\}$, we have $\mathcal{L}=\mathcal{L}_{0}$ is a Lie algebra and if $\Lambda=\mathbb{Z}_{2}$ and $\epsilon(\overline{1}, \overline{1})=-1$, then $\mathcal{L}$ is a Lie superalgebra). Clearly, Hom-Lie algebras and Lie color algebras are examples of Hom-Lie color algebras.

Let $\mathcal{L}$ be a Hom-Lie color algebra over the base field $\mathbb{F}$, and let $-: \mathbb{F} \longrightarrow \mathbb{F}$ be an involutive automorphism which we call a conjugation on $\mathbb{F}$. An involution is a conjugate-linear map $*: \mathcal{L} \longrightarrow \mathcal{L} ;\left(x \longmapsto x^{*}\right)$, such that $*^{2}=*,[x, y]^{*}=\left[y^{*}, x^{*}\right]$ and $(\phi(x))^{*}=\phi\left(x^{*}\right)$, for all $x, y \in \mathcal{L}$.

Definition 2.3. A regular Hom-Lie color algebra endowed with an involution is said to be an involutive regular Hom-Lie color algebra. An involutive subset of an involutive algebra is a subset globally invariant by the involution.

The usual regularity conditions will be understood in the involutive graded sense. For instance, a subalgebra $A$ of $\mathcal{L}$ is an involutive graded space $A=\bigoplus_{\lambda \in \Lambda} A^{\lambda}$ of $\mathcal{L}$ such that $[A, A] \subset A$ and $\phi(A)=A$. An involutive graded subspace $I=\bigoplus_{\lambda \in \Lambda} I^{\lambda}$ of $\mathcal{L}$ is called an ideal if $[I, \mathcal{L}] \subset I$ and $\phi(I)=I$. We say that $\mathcal{L}$ is simple if $[\mathcal{L}, \mathcal{L}] \neq 0$ and its only (involutive graded) ideals are $(0)$ and $\mathcal{L}$. 
From now on, $(\mathcal{L}, *)$ denotes an involutive regular Hom-Lie color algebra. We introduce the concept of split involutive regular Hom-Lie color algebra in an analogous way. We begin by considering a maximal involutive abelian graded subalgebra $\mathcal{H}=\bigoplus_{\lambda \in \Lambda} \mathcal{H}^{\lambda}$ among the involutive abelian graded subalgebras of $\mathcal{L}$. Note that $\mathcal{H}$ is necessarily a maximal involutive abelian subalgebra of $\mathcal{L}$ (see [7, Lemma 2.4]). Let us introduce the class of split algebras in the framework of involutive split regular Hom-Lie color algebras. We denote by $\mathcal{H}=\bigoplus_{\lambda \in \Lambda} \mathcal{H}^{\lambda}$ a maximal involutive abelian subalgebra of $(\mathcal{L}, *)$. For a linear functional

$$
\alpha:\left(\mathcal{H}^{0}, *\right) \longrightarrow(\mathbb{F},-),
$$

that is $\alpha\left(h^{*}\right)=\overline{\alpha(h)}$ for any $h \in \mathcal{H}^{0}$, we define the root space of $\mathcal{L}$ (with respect to $\mathcal{H}$ ) associated to $\alpha$ as the subspace

$$
\mathcal{L}_{\alpha}:=\left\{x_{\alpha} \in \mathcal{L} \mid\left[h^{0}, x_{\alpha}\right]=\alpha\left(h^{0}\right) \phi\left(x_{\alpha}\right) \text { for all } h^{0} \in \mathcal{H}^{0}\right\} .
$$

The elements $\alpha:\left(\mathcal{H}^{0}, *\right) \longrightarrow(\mathbb{F},-)$ satisfying $\mathcal{L}_{\alpha} \neq\{0\}$ are called roots of $\mathcal{L}$ with respect to $\mathcal{H}$. We denote by $\Pi:=\left\{\alpha \in(\mathcal{H} \backslash\{0\}, *) \longrightarrow(\mathbb{F},-) \mid \mathcal{L}_{\alpha} \neq\{0\}\right\}$.

Definition 2.4. We say that $\mathcal{L}$ is a split involutive regular Hom-Lie color algebra, with respect to $\mathcal{H}$, if

$$
\mathcal{L}=\mathcal{H} \oplus\left(\bigoplus_{\alpha \in \Pi} \mathcal{L}_{\alpha}\right)
$$

We also say that $\Pi$ is the root system of $\mathcal{L}$.

Observe that we have $\mathcal{H}^{*}=\mathcal{H}$. For convenience, the mappings $\left.\phi\right|_{\mathcal{H}},\left.\phi\right|_{\mathcal{H}}{ }^{-1}$ : $\mathcal{H} \longrightarrow \mathcal{H}$ will be denoted by $\phi$ and $\phi^{-1}$ respectively.

Lemma 2.5. Let $\mathcal{L}=\bigoplus_{\lambda \in \Lambda} \mathcal{L}^{\lambda}$ be a split involutive regular Hom-Lie color algebra, with root space decomposition $\mathcal{L}=\mathcal{H} \oplus\left(\bigoplus_{\alpha \in \Pi} \mathcal{L}_{\alpha}\right)$. Then

(1) $\mathcal{L}_{0}=\mathcal{H}$;

(2) for any $\alpha \in \Pi \cup\{0\}$, we have $\mathcal{L}_{\alpha}=\bigoplus_{\lambda \in \Lambda} \mathcal{L}_{\alpha}^{\lambda}$, where $\mathcal{L}_{\alpha}^{\lambda}=\mathcal{L}^{\lambda} \cap \mathcal{L}_{\alpha}$;

(3) $\mathcal{H}^{\lambda}=\mathcal{L}_{0}^{\lambda}\left(\right.$ in particular, $\left.\mathcal{H}^{0}=\mathcal{L}_{0}^{0}\right)$;

(4) $\mathcal{L}^{0}$ is a split Hom-Lie algebra, with respect to $\mathcal{H}^{0}=\mathcal{L}_{0}^{0}$, with root space decomposition $\mathcal{L}^{0}=\mathcal{L}_{0}^{0} \oplus\left(\bigoplus_{\alpha \in \Pi} \mathcal{L}_{\alpha}^{0}\right)$.

Proof. It is a consequence of Lemma 2.6 in [7].

Note that if $\mathcal{L}$ is a split involutive regular Hom-Lie color algebra, with root space decomposition $\mathcal{L}=\mathcal{H} \oplus\left(\bigoplus_{\alpha \in \Pi} \mathcal{L}_{\alpha}\right)$, taking into account Lemma 2.5, we then write

$$
\mathcal{L}=\bigoplus_{\lambda \in \Lambda}\left(\mathcal{H}^{\lambda} \oplus\left(\bigoplus_{\alpha \in \Pi} \mathcal{L}_{\alpha}^{\lambda}\right)\right)=\mathcal{L}_{0}^{0} \oplus\left(\bigoplus_{\lambda \in \Lambda} \bigoplus_{\alpha \in \Pi} \mathcal{L}_{\alpha}^{\lambda}\right)
$$

We denote by $\Pi^{\lambda}$ the set $\left\{\alpha \in \Pi \mid \mathcal{L}_{\alpha}^{\lambda} \neq 0\right\}$, for any $\lambda \in \Lambda$.

Lemma 2.6. For any $\alpha, \beta \in \Pi \cup\{0\}$ and any $\lambda \in \Lambda$, the following assertions hold:

(1) $\phi\left(\mathcal{L}_{\alpha}^{\lambda}\right) \subset \mathcal{L}_{\alpha \phi^{-1}}^{\lambda}$ and $\phi^{-1}\left(\mathcal{L}_{\alpha}^{\lambda}\right) \subset \mathcal{L}_{\alpha \phi}^{\lambda}$;

(2) $\left[\mathcal{L}_{\alpha}^{\lambda}, \mathcal{L}_{\beta}^{\mu}\right] \subset \mathcal{L}_{(\alpha+\beta) \phi^{-1}}^{\lambda+\mu}$;

(3) $\left(\mathcal{L}_{\alpha}^{\lambda}\right)^{*}=\mathcal{L}_{-\alpha}^{-\lambda}$. 
Proof. (1) By Lemma 2.5 and the fact that $\phi$ is an automorphism, we have

$$
\begin{aligned}
\phi\left(\mathcal{L}_{\alpha}^{\lambda}\right) & =\phi\left(\mathcal{L}^{\lambda} \cap \mathcal{L}_{\alpha}\right) \\
& =\phi\left(\mathcal{L}_{\alpha}\right) \cap\left(\mathcal{L}^{\lambda}\right) .
\end{aligned}
$$

Now taking into account Lemma 2.7 in [7], from (2.1) we get

$$
\phi\left(\mathcal{L}_{\alpha}^{\lambda}\right) \subset \mathcal{L}_{\alpha \phi^{-1}} \cap \mathcal{L}^{\lambda}=\mathcal{L}_{\alpha \phi^{-1}}^{\lambda}
$$

In a similar way, one gets the second statement in (1).

(2) For any $h^{0} \in \mathcal{H}^{0}, x_{\alpha}^{\lambda} \in \mathcal{L}_{\alpha}^{\lambda}$, and $y_{\beta}^{\mu} \in \mathcal{L}_{\beta}^{\mu}$, by denoting $h^{\prime}=\phi\left(h^{0}\right)$, from the $\epsilon$-Hom-Jacobi identity we have

$$
\begin{aligned}
{\left[h^{\prime},\left[x_{\alpha}^{\lambda}, y_{\beta}^{\mu}\right]\right] } & =\left[\left[h^{0}, x_{\alpha}^{\lambda}\right], \phi\left(y_{\beta}^{\mu}\right)\right]+\epsilon\left(h^{0}, x_{\alpha}^{\lambda}\right)\left[\phi\left(x_{\alpha}^{\lambda}\right),\left[h^{0}, y_{\beta}^{\mu}\right]\right] \\
& =\left[\alpha\left(h^{0}\right) \phi\left(x_{\alpha}^{\lambda}\right), \phi\left(y_{\beta}^{\mu}\right)\right]+\beta\left(h^{0}\right)\left[\phi\left(x_{\alpha}^{\lambda}\right), \phi\left(y_{\beta}^{\mu}\right)\right] \\
& =(\alpha+\beta)\left(h^{0}\right) \phi\left(\left[x_{\alpha}^{\lambda}, y_{\beta}^{\mu}\right]\right) \\
& =(\alpha+\beta)\left(\phi^{-1}\left(h^{\prime}\right) \phi\left(\left[x_{\alpha}^{\lambda}, y_{\beta}^{\mu}\right]\right) .\right.
\end{aligned}
$$

Therefore, we get $\left[x_{\alpha}^{\lambda}, y_{\beta}^{\mu}\right] \in \mathcal{L}_{(\alpha+\beta) \phi^{-1}}^{\lambda+\mu}$ and so $\left[\mathcal{L}_{\alpha}^{\lambda}, \mathcal{L}_{\beta}^{\mu}\right] \subset \mathcal{L}_{(\alpha+\beta) \phi^{-1}}^{\lambda+\mu}$.

(3) From the fact that " $*$ " is an involutive automorphism and Lemma 2.3 in [21], we conclude the result.

Lemma 2.7. If $\alpha \in \Pi$ then $\alpha \phi^{-m} \in \Pi$ for any $m \in \mathbb{Z}$.

Proof. It is a directly consequence of Lemma 2.8 in [7].

Definition 2.8. A root system $\Pi$ of a split involutive regular Hom-Lie color algebra $\mathcal{L}$ is called symmetric if $\Pi=-\Pi$.

\section{Connections of Roots And Decompositions}

In the following, $\mathcal{L}$ denotes a split involutive regular Hom-Lie color algebra with a symmetric root system $\Pi$ and $\mathcal{L}=\mathcal{H} \oplus\left(\bigoplus_{\alpha \in \Pi} \mathcal{L}_{\alpha}\right)$, the corresponding root space decomposition. We begin by developing the techniques of connections of roots in the same setting as [3].

Definition 3.1. Let $\alpha, \beta$ be two nonzero roots in $\Pi$. We say that $\alpha$ is connected to $\beta$, denoted by $\alpha \sim \beta$, if there exists a family

$$
\left\{\alpha_{1}, \alpha_{2}, \alpha_{3}, \ldots, \alpha_{k}\right\} \subset \Pi,
$$

satisfying the following conditions:

If $k=1$ :

(1) $\alpha_{1} \in\left\{\alpha \phi^{-n}: n \in \mathbb{N}\right\} \cap\left\{ \pm \beta \phi^{-m}: m \in \mathbb{N}\right\}$.

If $k \geq 2$ :

(1) $\alpha_{1} \in\left\{\alpha \phi^{-n}: n \in \mathbb{N}\right\}$. 
(2) $\alpha_{1} \phi^{-1}+\alpha_{2} \phi^{-1} \in \Pi$,

$\alpha_{1} \phi^{-2}+\alpha_{2} \phi^{-2}+\alpha_{3} \phi^{-1} \in \Pi$,

$\alpha_{1} \phi^{-3}+\alpha_{2} \phi^{-3}+\alpha_{3} \phi^{-2}+\alpha_{4} \phi^{-1} \in \Pi$,

$\ldots$

$\alpha_{\phi}^{-i}+\alpha_{2} \phi^{-i}+\alpha_{3} \phi^{-i+1}+\cdots+\alpha_{i+1} \phi^{-1} \in \Pi$,

$\ldots$

$\alpha_{1} \phi^{-k+2}+\alpha_{2} \phi^{-k+2}+\alpha_{3} \phi^{-k+3}+\cdots+\alpha_{i} \phi^{-k+i}+\cdots+\alpha_{k-1} \phi^{-1} \in \Pi$.

(3) $\alpha_{1} \phi^{-k+1}+\alpha_{2} \phi^{-k+1}+\alpha_{3} \phi^{-k+2}+\cdots+\alpha_{i} \phi^{-k+i-1}+\cdots+\alpha_{k} \phi^{-1} \in\left\{ \pm \beta \phi^{-m}\right.$ : $m \in \mathbb{N}\}$.

The family $\left\{\alpha_{1}, \alpha_{2}, \alpha_{3}, \ldots, \alpha_{k}\right\} \subset \Pi$ is called a connection from $\alpha$ to $\beta$.

Note that the case $k=1$ in Definition 3.1 is equivalent to the fact that $\beta=\varepsilon \alpha \phi^{z}$ for some $z \in \mathbb{Z}$ and $\varepsilon \in\{ \pm 1\}$.

Lemma 3.2. The following assertions hold:

(1) For any $\alpha \in \Pi$, we have that $\alpha \phi^{z_{1}}$ is connected to $\alpha \phi^{z_{2}}$ for every $z_{1}, z_{2} \in \mathbb{Z}$. We also have that $\alpha \phi^{z_{1}}$ is connected to $-\alpha \phi^{z_{2}}$ in case $-\alpha \phi^{z_{2}} \in \Pi$.

(2) Let $\left\{\alpha_{1}, \alpha_{2}, \alpha_{3}, \ldots, \alpha_{k}\right\}$ be a connection from $\alpha$ to $\beta$. Suppose that $\alpha_{1}=$ $\alpha \phi^{-n}, n \in \mathbb{N}$. Then for any $r \in \mathbb{N}$ such that $r \geq n$, there exists a connection $\left\{\tilde{\alpha}_{1}, \tilde{\alpha}_{2}, \ldots, \tilde{\alpha}_{k}\right\}$ from $\alpha$ to $\beta$ such that $\tilde{\alpha}_{1}=\alpha \phi^{-r}$.

(3) Let $\left\{\alpha_{1}, \alpha_{2}, \alpha_{3}, \ldots, \alpha_{k}\right\}$ be a connection from $\alpha$ to $\beta$. Suppose that $\alpha_{1}=$ $\varepsilon \beta \phi^{-m}, m \in \mathbb{N}$ in case $k=1$ or

$$
\alpha_{1} \phi^{-k+1}+\alpha_{2} \phi^{-k+1}+\alpha_{3} \phi^{-k+2}+\cdots+\alpha_{k} \phi^{-1}=\varepsilon \beta \phi^{-m},
$$

in case $k \geq 2$, with $\varepsilon \in\{ \pm 1\}$. Then for any $r \in \mathbb{N}$ such that $r \geq m$, there exists a connection $\left\{\tilde{\alpha}_{1}, \tilde{\alpha}_{2}, \ldots, \tilde{\alpha}_{k}\right\}$ from $\alpha$ to $\beta$ such that $\tilde{\alpha}_{1}=\varepsilon \beta \phi^{-r}$ in case $k=1$ or

$$
\tilde{\alpha}_{1} \phi^{-k+1}+\tilde{\alpha}_{2} \phi^{-k+1}+\tilde{\alpha}_{3} \phi^{-k+2}+\cdots+\tilde{\alpha}_{k} \phi^{-1}=\varepsilon \beta \phi^{-r},
$$

in case $k \geq 2$.

Proof. The assertions are proved in [3, Lemmas 2.2 and 2.3].

Proposition $3.3([3])$. The relation $\sim$ in $\Pi$ defined by

$$
\lambda \sim \mu \text { if and only if } \lambda \text { is connected to } \mu
$$

is an equivalence relation.

Proof. The proof is virtually identical to the proof of [3, Proposition 2.4].

By the above proposition, we can consider the equivalence relation in $\Pi$ by the connection relation $\sim$ in $\Pi$. So we denote by

$$
\Pi / \sim:=\{[\alpha]: a \in \Pi\},
$$

where $[\alpha]$ denotes the set of nonzero roots of $\mathcal{L}$ which are connected to $\alpha$. Clearly, if $\beta \in[\alpha]$ then $-\beta \in[\alpha]$ and by Proposition 3.3 if $\beta \notin[\alpha]$ then $[\alpha] \cap[\beta]=\emptyset$. 
Our next goal in this section is to associate an adequate ideal $I_{[\alpha]}$ of $\mathcal{L}$ to any $[\alpha]$. For a fixed $\alpha \in \Pi$, we define

$$
I_{0,[\alpha]}:=\operatorname{span}_{\mathbb{F}}\left\{\left[\mathcal{L}_{\beta},\left(\mathcal{L}_{\beta}\right)^{*}\right]: \beta \in[\alpha]\right\} \subset \mathcal{H} .
$$

Then $I_{0,[\alpha]}$ is the direct sum of

$$
\sum_{\beta \in[\alpha], \lambda \in \Lambda}\left[\mathcal{L}_{\beta}^{\lambda}, \mathcal{L}_{-\beta}^{-\lambda}\right] \subset \mathcal{L}_{0}^{0} \quad \text { and } \sum_{\beta \in[\alpha], \lambda, \mu \in \Lambda, \lambda+\mu \neq 0}\left[\mathcal{L}_{\beta}^{\lambda}, \mathcal{L}_{-\beta}^{\mu}\right] \subset \bigoplus_{\lambda \in \Lambda \backslash\{0\}} \mathcal{H}^{\lambda} .
$$

Next, we define

$$
\mathcal{V}_{[\alpha]}:=\bigoplus_{\beta \in[\alpha]} \mathcal{L}_{\beta}=\bigoplus_{\lambda \in \Lambda} \bigoplus_{\beta \in[\alpha]} \mathcal{L}_{\beta}^{\lambda} .
$$

Finally, we denote by $I_{[\alpha]}$ the direct sum of the two graded subspaces above, that is,

$$
I_{[\alpha]}:=I_{0,[\alpha]} \oplus \mathcal{V}_{[\alpha]} .
$$

Proposition 3.4. For any $\alpha \in \Pi$, the graded subspace $I_{[\alpha]}$ is an ivolutive subalgebra of $\mathcal{L}$.

Proof. First, we are going to check that $I_{[\alpha]}$ satisfies $\left[I_{[\alpha]}, I_{[\alpha]}\right] \subset I_{[\alpha]}$. By the fact that $\mathcal{L}_{0}=\mathcal{H}$ and $\left(3.1\right.$, it is clear that $\left[I_{0,[\alpha]}, I_{0,[\alpha]}\right]=0$, and we have

$$
\begin{aligned}
{\left[I_{[\alpha]}, I_{[\alpha]}\right] } & =\left[I_{0,[\alpha]} \oplus \mathcal{V}_{[\alpha]}, I_{0,[\alpha]} \oplus \mathcal{V}_{[\alpha]}\right] \\
& \subset\left[I_{0,[\alpha]}, \mathcal{V}_{[\alpha]}\right]+\left[\mathcal{V}_{[\alpha]}, I_{0,[\alpha]}\right]+\left[\mathcal{V}_{[\alpha]}, \mathcal{V}_{[\alpha]}\right]
\end{aligned}
$$

Let us consider the first summand in 3.2 . Note that by 3.1 and the fact that $\mathcal{L}_{0}=\mathcal{H}$ we have

$$
\left[I_{0,[\alpha]}, \mathcal{V}_{[\alpha]}\right]=\left[I_{0,[\alpha]}, \bigoplus_{\beta \in[\alpha]} \mathcal{L}_{\beta}\right] \subset \sum_{\beta \in[\alpha]}\left[\mathcal{L}_{0}, \mathcal{L}_{\beta}\right]
$$

Given $\beta \in[\alpha]$, by Lemma $2.6+(2)$, one gets $\left[I_{0,[\alpha]}, \mathcal{L}_{\beta}\right] \subset \mathcal{L}_{\beta \phi^{-1}}$, where $\beta \phi^{-1} \in[\alpha]$. Hence,

Similarly, we can also get

$$
\left[I_{0,[\alpha]}, \mathcal{V}_{[\alpha]}\right] \subset \mathcal{V}_{[\alpha]}
$$

$$
\left[\mathcal{V}_{[\alpha]}, I_{0,[\alpha]}\right] \subset \mathcal{V}_{[\alpha]}
$$

Consider now the third summand in 3.2 . We have

$$
\left[\mathcal{V}_{[\alpha]}, \mathcal{V}_{[\alpha]}\right] \subset \sum_{\beta, \gamma \in[\alpha]}\left[\mathcal{L}_{\beta}, \mathcal{L}_{\gamma}\right]
$$

Given $\beta, \gamma \in[\alpha]$ such that $0 \neq\left[\mathcal{L}_{\beta}, \mathcal{L}_{\gamma}\right]$. If $\gamma=-\beta$, we have

$$
\left[\mathcal{L}_{\beta}, \mathcal{L}_{\gamma}\right]=\left[\mathcal{L}_{\beta}, \mathcal{L}_{-\beta}\right]=\left[\mathcal{L}_{\beta},\left(\mathcal{L}_{\beta}\right)^{*}\right] \subset I_{0,[\alpha]} .
$$

Suppose that $0 \neq \beta+\gamma$. By Lemma 2.6 $(2)$, one gets $(\beta+\gamma) \phi^{-1} \in \Pi$. Therefore, we get $\{\beta, \gamma\}$ a connection from $\beta$ to $(\beta+\gamma) \phi^{-1}$. The equivalence relation $\sim$ gives us $(\beta+\gamma) \phi^{-1} \in[\alpha]$ and so $\left[\mathcal{L}_{\beta}, \mathcal{L}_{\gamma}\right] \subset \mathcal{L}_{(\beta+\gamma) \phi^{-1}} \subset \mathcal{V}_{[\alpha]}$. Hence,

$$
\left[\mathcal{V}_{[\alpha]}, \mathcal{V}_{[\alpha]}\right]=\left[\bigoplus_{\beta \in[\alpha]} \mathcal{L}_{\beta}, \bigoplus_{\beta \in[\alpha]} \mathcal{L}_{\beta}\right] \subset I_{0,[\alpha]} \oplus \mathcal{V}_{[\alpha]}
$$


From 3.3, 3.4, and 3.5, we conclude that $\left[I_{[\alpha]}, I_{[\alpha]}\right] \subset I_{[\alpha]}$.

Second, we have to verify that $\phi\left(I_{[\alpha]}\right)=I_{[\alpha]}$. But this is a direct consequence of Lemma 2.6 (1) and Lemma 3.2 (1).

Third, we must show that $I_{[\alpha]}=\left(I_{[\alpha]}\right)^{*}$. It is easy to see that $I_{0,[\alpha]}=\left(I_{0,[\alpha]}\right)^{*}$ and $\mathcal{V}_{[\alpha]}=\left(\mathcal{V}_{[\alpha]}\right)^{*}$, thanks to the given definition. Now taking into account $I_{[\alpha]}=$ $I_{0,[\alpha]} \oplus \mathcal{V}_{[\alpha]}$, we get $I_{[\alpha]}=\left(I_{[\alpha]}\right)^{*}$.

Proposition 3.5. If $[\alpha] \neq[\beta]$, then $\left[I_{[\alpha]}, I_{[\beta]}\right]=0$.

Proof. We have

$$
\begin{aligned}
{\left[I_{[\alpha]}, I_{[\beta]}\right] } & =\left[I_{0,[\alpha]} \oplus \mathcal{V}_{[\alpha]}, I_{0,[\beta]} \oplus \mathcal{V}_{[\beta]}\right] \\
& \subset\left[I_{0,[\alpha]}, \mathcal{V}_{[\beta]}\right]+\left[\mathcal{V}_{[\alpha]}, I_{0,[\beta]}\right]+\left[\mathcal{V}_{[\alpha]}, \mathcal{V}_{[\beta]}\right]
\end{aligned}
$$

Let us consider the third summand in (3.6) and suppose there exist $\eta \in[\alpha]$ and $\mu \in[\beta]$, such that $0 \neq\left[\mathcal{L}_{\eta}, \mathcal{L}_{\mu}\right]$. By the condition $[\alpha] \neq[\beta]$, one gets $\eta \neq-\mu$, then $(\eta+\mu) \phi^{-1} \in \Pi$. Hence, $\left\{\eta, \mu,-\eta \phi^{-1}\right\}$ is a connection from $\eta$ to $\mu$. By the transitivity of $\sim$, we have $\alpha \in[\beta]$, which is a contradiction. Therefore, $\left[\mathcal{L}_{\eta}, \mathcal{L}_{\mu}\right]=0$ and so

$$
\left[\mathcal{V}_{[\alpha]}, \mathcal{V}_{[\beta]}\right]=\{0\}
$$

Consider now the first summand in 3.6 and suppose there exist $\eta \in[\alpha]$ and $\mu \in[\beta]$ such that $0 \neq\left[\left[\mathcal{L}_{\eta},\left(\mathcal{L}_{\eta}\right)^{*}\right], \phi\left(\mathcal{L}_{\mu}\right)\right]$. Then

$$
\left[\left[\mathcal{L}_{\eta}^{\lambda}, \mathcal{L}_{-\eta}^{-\lambda}\right], \phi\left(\mathcal{L}_{\mu}\right)\right] \neq 0
$$

for some $\lambda \in \Lambda$. By the $\epsilon$-Hom-Jacobi identity, we have either $\left[\mathcal{L}_{\eta}^{\lambda}, \phi\left(\mathcal{L}_{\mu}\right)\right] \neq 0$ or $\left[\mathcal{L}_{-\eta}^{-\lambda}, \phi\left(\mathcal{L}_{\mu}\right)\right] \neq 0$, and so we get $\left[\mathcal{V}_{[\alpha]}, \mathcal{V}_{[\beta]}\right] \neq 0$ in any case, which is a contradiction to 3.7 . Hence,

In a similar way, we also have

$$
\left[I_{0,[\alpha]}, \mathcal{V}_{[\beta]}\right]=0
$$

$$
\left.\left[\mathcal{V}_{[\alpha]}, I_{0,[\beta]}\right]\right]=0 \text {. }
$$

Finally, from $(3.7), 3.8)$, and $(3.9)$, we conclude that $\left[I_{[\alpha]}, I_{[\beta]}\right]=0$.

Theorem 3.6. The following assertions hold:

(1) For any $\alpha \in \Pi$, the involutive Hom-Lie color subalgebra

$$
I_{[\alpha]}=I_{0,[\alpha]} \oplus \mathcal{V}_{[\alpha]}
$$

of $\mathcal{L}$ associated to $[\alpha]$ is an involutive ideal of $\mathcal{L}$.

(2) If $\mathcal{L}$ is simple, then there exists a connection from $\alpha$ to $\beta$ for any $\alpha, \beta \in \Pi$ and $\mathcal{H}=\sum_{\alpha \in \Pi}\left[\mathcal{L}_{\alpha},\left(\mathcal{L}_{\alpha}\right)^{*}\right]$.

Proof. (1) Since $\left[I_{[\alpha]}, \mathcal{H}\right]=\left[I_{[\alpha]}, \mathcal{L}_{0}\right] \subset \mathcal{V}_{[\alpha]}$, taking into account Proposition 3.4 and Proposition 3.5 , we have

$$
\left[I_{[\alpha]}, \mathcal{L}\right]=\left[I_{[\alpha]}, \mathcal{H} \oplus\left(\bigoplus_{\beta \in[\alpha]} \mathcal{L}_{\beta}\right) \oplus\left(\bigoplus_{\gamma \notin[\alpha]} \mathcal{L}_{\gamma}\right)\right] \subset I_{[\alpha]} .
$$

As we also have by Proposition 3.4 that $\phi\left(I_{[\alpha]}\right)=I_{[\alpha]}$ and $I_{[\alpha]}=\left(I_{[\alpha]}\right)^{*}$, we conclude that $I_{[\alpha]}$ is an involutive ideal of $\mathcal{L}$. 
(2) The simplicity of $\mathcal{L}$ implies $I_{[\alpha]}=\mathcal{L}$. From here, it is clear that $[\alpha]=\Pi$ and $\mathcal{H}=\sum_{\alpha \in \Pi}\left[\mathcal{L}_{\alpha},\left(\mathcal{L}_{\alpha}\right)^{*}\right]$.

Theorem 3.7. For a vector space complement $\mathcal{U}$ of $\operatorname{span}_{\mathbb{F}}\left\{\left[\mathcal{L}_{\alpha},\left(\mathcal{L}_{\alpha}\right)^{*}\right]: \alpha \in \Pi\right\}$ in $\mathcal{H}$, we have

$$
\mathcal{L}=\mathcal{U} \oplus \sum_{[\alpha] \in \Pi / \sim} I_{[\alpha]}
$$

where any $I_{[\alpha]}$ is one of the involutive ideals of $\mathcal{L}$ described in Theorem 3.6. (1), satisfying $\left[I_{[\alpha]}, I_{[\beta]}\right]=0$, whenever $[\alpha] \neq[\beta]$.

Proof. Each $I_{[\alpha]}$ is well defined and by Theorem 3.6-(1), an involutive ideal of $\mathcal{L}$. It is clear that

$$
\mathcal{L}=\mathcal{H} \oplus\left(\bigoplus_{\alpha \in \Pi} \mathcal{L}_{\alpha}\right)=\mathcal{U} \oplus \sum_{[\alpha] \in \Pi / \sim} I_{[\alpha]} .
$$

Finally, Proposition 3.5 gives us $\left[I_{[\alpha]}, I_{[\beta]}\right]=\{0\}$, if $[\alpha] \neq[\beta]$.

Let us denote by $Z(\mathcal{L})$ the center of $\mathcal{L}$, that is, $Z(\mathcal{L})=\{x \in \mathcal{L}:[x, \mathcal{L}]=0\}$.

Definition 3.8. A Hom-Lie color algebra $\mathcal{L}$ is called perfect if $Z(\mathcal{L})=0$ and $[\mathcal{L}, \mathcal{L}]=\mathcal{L}$.

Corollary 3.9. If $\mathcal{L}$ is a perfect split involutive regular Hom-Lie color algebra, then $\mathcal{L}$ is the direct sum of the involutive ideals given in Theorem 3.6. (1),

$$
\mathcal{L}=\bigoplus_{[\alpha] \in \Pi / \sim} I_{[\alpha]}
$$

Proof. From $[\mathcal{L}, \mathcal{L}]=\mathcal{L}$, it is clear that $\mathcal{L}=\sum_{[\alpha] \in \Pi / \sim} I_{[\alpha]}$. Now, by $Z(\mathcal{L})=0$ and Proposition 3.5 the direct character of the sum is clear.

\section{The SIMPle COMPONEnts}

In this section, we focus on the simplicity of split involutive regular Hom-Lie color algebras $\mathcal{L}$ by centering our attention in those of maximal length.

Lemma 4.1. Let $\mathcal{L}=\mathcal{H} \oplus\left(\bigoplus_{\alpha \in \Pi} \mathcal{L}_{\alpha}\right)$ be a split involutive regular Hom-Lie color algebra. If $I$ is an ideal of $\mathcal{L}$, then $I=(I \cap \mathcal{H}) \oplus\left(\bigoplus_{\alpha \in \Pi}\left(I \cap \mathcal{L}_{\alpha}\right)\right)$.

Proof. It is analogous to the proof of Lemma 4.1 in [7].

Taking into account the above lemma, observe that the grading of $I$ together with Lemma 2.5 (2) allow us to assert that

$$
I=\bigoplus_{\lambda \in \Lambda} I^{\lambda}=\bigoplus_{\lambda \in \Lambda}\left(\left(I^{\lambda} \cap \mathcal{H}^{\lambda}\right) \oplus\left(\bigoplus_{\alpha \in \Pi_{I}}\left(I^{\lambda} \cap \mathcal{L}_{\alpha}^{\lambda}\right)\right)\right)
$$

where $\Pi_{I}:=\left\{\alpha \in \Pi: I^{\lambda} \cap \mathcal{L}_{\alpha}^{\lambda} \neq 0, \forall \lambda \in \Lambda\right\}$.

Lemma 4.2. Let $\mathcal{L}$ be a centerless split involutive regular Hom-Lie color algebra. If $I$ is an ideal of $\mathcal{L}$ such that $I \subset \mathcal{H}$, then $I=(0)$.

Proof. See [7, Lemma 4.2]. 
Let us introduce the concepts of root-multiplicativity and maximal length in the framework of split involutive regular Hom-Lie color algebras, in a similar way to the ones for split regular Hom-Lie color algebras in [7].

Definition 4.3. We say that a split involutive regular Hom-Lie color algebra $\mathcal{L}$ is root-multiplicative if given $\alpha \in \Pi^{\lambda}$ and $\beta \in \Pi^{\mu}$, with $\lambda, \mu \in \Lambda$, such that $\alpha+\beta \in \Pi$, then $\left[\mathcal{L}_{\alpha}^{\lambda}, \mathcal{L}_{\beta}^{\mu}\right] \neq 0$.

Definition 4.4. We say that a split involutive regular Hom-Lie color algebra $\mathcal{L}$ is of maximal length if for any $\alpha \in \Pi^{\lambda}$ with $\lambda \in \Lambda$, we have $\operatorname{dim} \mathcal{L}_{k \alpha}^{k \lambda}=1$ for $k \in\{ \pm 1\}$.

Observe that for a split involutive regular Hom-Lie color algebra $\mathcal{L}$ of maximal length, 4.1 allows us to assert that given any nonzero graded ideal $I$ of $\mathcal{L}$ we can write

$$
I=\bigoplus_{\lambda \in \Lambda}\left(\left(I^{\lambda} \cap \mathcal{H}^{\lambda}\right) \oplus\left(\bigoplus_{\alpha \in \Pi_{I}^{\lambda}} \mathcal{L}_{\alpha}^{\lambda}\right)\right),
$$

where $\Pi_{I}^{\lambda}:=\left\{\alpha \in \Pi: I^{\lambda} \cap \mathcal{L}_{\alpha}^{\lambda} \neq 0\right\}$ for each $\lambda \in \Lambda$.

Theorem 4.5. Let $\mathcal{L}$ be a perfect split involutive regular Hom-Lie color algebra of maximal length and root-multiplicative. If $\mathcal{L}$ has all of its nonzero roots connected, then any ideal $I$ of $\mathcal{L}$ satisfies $I^{\star}=I$.

Proof. Consider any nonzero ideal $I$ of $\mathcal{L}$. By 4.2 and Lemma 4.2, we have $I=\bigoplus_{\lambda \in \Lambda}\left(\left(I^{\lambda} \cap \mathcal{H}^{\lambda}\right) \oplus\left(\bigoplus_{\alpha \in \Pi_{I}^{\lambda}} \mathcal{L}_{\alpha}^{\lambda}\right)\right)$, with $\Pi_{I}^{\lambda} \subset \Pi^{\lambda}$ for any $\lambda \in \Lambda$ and some $\Pi_{I}^{\lambda} \neq \phi$. Let us fix some $\alpha_{0} \in \Pi_{I}^{\lambda}$, so that

$$
0 \neq \mathcal{L}_{\alpha_{0}}^{\lambda} \subset I
$$

The fact that $\phi(I)=I$ together with Lemma 2.6-(2) allows us to assert that

$$
\text { If } \alpha \in \Pi_{I} \text { then }\left\{\alpha \phi^{z}: z \in \mathbb{Z}\right\} \subset \Pi_{I},
$$

that is,

$$
\left\{\mathcal{L}_{a_{0} \phi^{z}}^{\lambda}: z \in \mathbb{Z}\right\} \subset I .
$$

Taking into account the facts that $[\mathcal{L}, \mathcal{L}]=\mathcal{L}$ and Corollary 3.9 imply $\mathcal{H}=$ $\sum_{\beta \in \Pi}\left[\mathcal{L}_{\beta},\left(\mathcal{L}_{\beta}\right)^{*}\right]$, the grading of $\mathcal{L}$ gives us

$$
\mathcal{H}^{0}=\sum_{\lambda \in \Lambda, \beta \in \Pi}\left[\mathcal{L}_{\beta}^{\lambda}, \mathcal{L}_{-\beta}^{-\lambda}\right]
$$

From here, since $\alpha_{0} \neq 0$, there exist $\beta \in \Pi$ and $\mu \in \Lambda$ such that $\alpha_{0}\left(\left[\mathcal{L}_{\beta}^{\lambda}, \mathcal{L}_{-\beta}^{-\lambda}\right]\right) \neq 0$. If $\beta \in\left\{ \pm \alpha_{0} \phi^{z}: z \in \mathbb{Z}\right\}$ as $0 \neq\left[\mathcal{L}_{a_{0} \phi^{z}}^{\lambda}, \mathcal{L}_{-a_{0} \phi^{z}}^{-\lambda}\right] \subset I$, then

$$
\mathcal{L}_{-a_{0}}=-\alpha_{0}\left(\left[\mathcal{L}_{a_{0} \phi^{z}}^{\lambda}, \mathcal{L}_{-a_{0} \phi^{z}}^{-\lambda}\right]\right)^{-1}\left[\left[\mathcal{L}_{a_{0} \phi^{z}}^{\lambda}, \mathcal{L}_{-a_{0} \phi^{z}}^{-\lambda}\right], \mathcal{L}_{-\alpha_{0}}^{\lambda}\right] \subset I .
$$

So in this case, we obtain $\mathcal{L}_{-a_{0}}=\left(\mathcal{L}_{a_{0}}\right)^{*} \subset I$.

Now, let us take any $\beta \in \Pi$ such that $\beta \notin\left\{ \pm \alpha_{0} \phi^{z}: z \in \mathbb{Z}\right\}$. Since $\alpha_{0}$ and $\beta$ are connected, we have a connection $\left\{\alpha_{1}, \alpha_{2}, \ldots, \alpha_{k}\right\}, k \geq 2$, from $\alpha_{0}$ to $\beta$ satisfying 
the following conditions:

$$
\begin{aligned}
& \alpha_{1}=\alpha_{0} \phi^{-n} \text { for some } n \in \mathbb{N} \text {, and } \\
& \alpha_{1} \phi^{-1}+\alpha_{2} \phi^{-1} \in \Pi, \\
& \alpha_{1} \phi^{-2}+\alpha_{2} \phi^{-2}+\alpha_{3} \phi^{-1} \in \Pi, \\
& \alpha_{1} \phi^{-3}+\alpha_{2} \phi^{-3}+\alpha_{3} \phi^{-2}+\alpha_{4} \phi^{-1} \in \Pi, \\
& \ldots \\
& \alpha_{\phi}^{-i}+\alpha_{2} \phi^{-i}+\alpha_{3} \phi^{-i+1}+\cdots+\alpha_{i+1} \phi^{-1} \in \Pi, \\
& \cdots \\
& \alpha_{1} \phi^{-k+2}+\alpha_{2} \phi^{-k+2}+\alpha_{3} \phi^{-k+3}+\cdots+\alpha_{i} \phi^{-k+i}+\cdots+\alpha_{k-1} \phi^{-1} \in \Pi, \\
& \alpha_{1} \phi^{-k+1}+\alpha_{2} \phi^{-k+1}+\alpha_{3} \phi^{-k+2}+\cdots+\alpha_{i} \phi^{-k+i-1}+\cdots+\alpha_{k} \phi^{-1}=\varepsilon \beta \phi^{-m}, \\
& \quad \text { for some } m \in \mathbb{N} \text { and } \varepsilon \in\{ \pm 1\} .
\end{aligned}
$$

Consider $\alpha_{1}, \alpha_{2} \in \Pi$ and $\alpha_{1}+\alpha_{2} \in \Pi$. Since $\alpha_{2} \in \Pi$, there exists $\lambda_{2} \in \Lambda$ such that $\mathcal{L}_{\alpha_{2}}^{\lambda_{2}} \neq 0$. From here, the root-multiplicativity and maximal length of $\mathcal{L}$ allow us to get

$$
0 \neq\left[\mathcal{L}_{\alpha_{1}}^{\lambda}, \mathcal{L}_{\alpha_{2}}^{\lambda_{2}}\right]=\mathcal{L}_{\left(\alpha_{1}+\alpha_{2}\right) \phi^{-1}}^{\lambda+\lambda_{2}}
$$

Since $0 \neq \mathcal{L}_{\alpha_{1}}^{\lambda} \subset I$ as consequence of 4.3 , we have

$$
0 \neq \mathcal{L}_{\left(\alpha_{1}+\alpha_{2}\right) \phi^{-1}}^{\lambda+\lambda_{2}} \subset I .
$$

We can argue in a similar way from $\alpha_{1} \phi^{-1}+\alpha_{2} \phi^{-1}, \alpha_{3}$ and $\left(\alpha_{1} \phi^{-1}+\alpha_{2} \phi^{-1}\right) \phi^{-1}+$ $\alpha_{3} \phi^{-1}=\alpha_{1} \phi^{-2}+\alpha_{2} \phi^{-2}+\alpha_{3} \phi^{-1} \in \Pi$ to get

$$
0 \neq \mathcal{L}_{\left(\alpha_{1}+\alpha_{2}\right) \phi^{-2}+\alpha_{3} \phi^{-1}}^{\lambda+\lambda_{2}+\lambda_{3}} \subset I, \quad \text { for some } \lambda_{3} \in \Lambda .
$$

If we follow this process with the connection $\left\{\alpha_{1}, \alpha_{2}, \ldots, \alpha_{k}\right\}$, then we obtain that

$$
0 \neq \mathcal{L}_{\alpha_{1} \phi^{-k+1}+\alpha_{2} \phi^{-k+1}+\alpha_{3} \phi^{-k+2}+\cdots+\alpha_{i} \phi^{-k+i-1}+\cdots+\alpha_{k} \phi^{-1}}^{\mu} \subset I, \quad \text { for some } \mu \in \Lambda,
$$

and so either $0 \neq \mathcal{L}_{\beta \phi^{-m}}^{\mu} \subset I$ or $0 \neq \mathcal{L}_{-\beta \phi^{-m}}^{-\mu} \subset I$, for some $\mu \in \Lambda$. That is,

$$
0 \neq \mathcal{L}_{\varepsilon \beta \phi^{-m}}^{\varepsilon \mu} \subset I, \quad \text { for some } \mu \in \Lambda, \varepsilon \in\{ \pm 1\} \text { and any } \beta \in \Pi \text {. }
$$

By Lemma 2.6-(1), we can get

$$
0 \neq \mathcal{L}_{\varepsilon \beta}^{\mu} \subset I, \quad \text { for some } \mu \in \Lambda, \varepsilon \in\{ \pm 1\},
$$

and for any $\beta \in \Pi$. Now taking into account (4.4), there exist $\alpha \in \Pi$ and $\eta \in \Lambda$ such that

$$
\left[\left[\mathcal{L}_{\alpha}^{\eta}, \mathcal{L}_{-\alpha}^{-\eta}\right], \phi\left(\mathcal{L}_{\varepsilon \beta}^{\mu}\right)\right] \neq 0 .
$$

By the $\epsilon$-Hom-Jacobi identity either $\left.\left[\mathcal{L}_{\alpha}^{\eta}, \phi\left(\mathcal{L}_{\varepsilon \beta}^{\mu}\right)\right] \neq 0\right]$ or $\left[\mathcal{L}_{-\alpha}^{-\eta}, \phi\left(\mathcal{L}_{\varepsilon \beta}^{\mu}\right)\right] \neq 0$ and so $0 \neq \mathcal{L}_{\alpha \phi^{-1}+\varepsilon \beta \phi^{-2}}^{\eta+\mu}$ or $0 \neq \mathcal{L}_{\alpha \phi^{-1}+\varepsilon \beta \phi^{-2}}^{-\eta+\mu}$. That is,

$$
0 \neq \mathcal{L}_{\kappa \alpha \phi^{-1}+\varepsilon \beta \phi^{-2}}^{\kappa \eta+\mu} \subset I
$$


for some $\kappa \in\{ \pm 1\}$. Since $\varepsilon \beta \in \Pi^{\mu}$, we have by the maximal length of $\mathcal{L}$ that $-\varepsilon \beta \in \Pi^{-\mu}$. From (4.6), the root-multiplicativity and maximal length of $\mathcal{L}$, we obtain

$$
0 \neq\left[\mathcal{L}_{\kappa \alpha \phi^{-1}+\varepsilon \beta \phi^{-2}}^{\kappa \eta+\mu}, \mathcal{L}_{-\varepsilon \beta \phi^{-2}}^{-\mu}\right]=\mathcal{L}_{\varepsilon \beta \phi^{-1}}^{\kappa \eta} \subset I .
$$

By Lemma 2.6 (1), we can get

$$
\mathcal{L}_{\kappa \alpha}^{\kappa \eta} \subset I .
$$

Taking into account 4.7) and (4.5) gives us

$$
\beta \phi^{-1}\left(\left[\mathcal{L}_{\alpha}^{\mu}, \mathcal{L}_{-\alpha}^{-\mu}\right]\right) \neq 0 .
$$

We have for any $\nu \in \Lambda$ such that $\mathcal{L}_{\varepsilon \beta}^{\nu} \neq 0$ necessarily

$$
\left.0 \neq\left[\mathcal{L}_{\alpha}^{\mu}, \mathcal{L}_{-\alpha}^{-\mu}\right], \phi\left(\mathcal{L}_{\varepsilon \beta}^{\nu}\right)\right]=\mathcal{L}_{\varepsilon \beta \phi^{-1}}^{\nu} \subset I,
$$

and so $\mathcal{L}_{\varepsilon \beta \phi^{-1}} \subset I$. That is, we can get $\mathcal{L}_{\varepsilon \beta} \subset I$ for any $\beta \in \Pi$ and some $\varepsilon \in\{ \pm 1\}$. In both cases we have

$$
0 \neq\left[\mathcal{L}_{\beta}^{\lambda},\left(\mathcal{L}_{\beta}^{\lambda}\right)^{*}\right] \subset I
$$

Hence,

$$
\left(\mathcal{L}_{\alpha_{0}}\right)^{*}=\mathcal{L}_{-a_{0}}=-\alpha_{0}\left(\left[\mathcal{L}_{\beta}^{\lambda}, \mathcal{L}_{-\beta}^{-\lambda}\right]\right)^{-1}\left[\left[\mathcal{L}_{\beta}^{\lambda}, \mathcal{L}_{-\beta}^{-\lambda}\right], \mathcal{L}_{-\alpha_{0}}\right] \subset I .
$$

Finally, the fact that $\mathcal{H}=\sum_{\beta \in \Pi}\left[\mathcal{L}_{\beta},\left(\mathcal{L}_{\beta}\right)^{*}\right]$ implies that

$$
\mathcal{H} \subset I \text {. }
$$

In particular, as $\mathcal{H}^{*}=\mathcal{H}$, we get $\left(\mathcal{H}^{\lambda} \cap I^{\lambda}\right)^{*}=\mathcal{H}^{\lambda} \cap I^{\lambda}$. From here and taking into account $\left(\bigoplus_{\alpha \in \Pi, \lambda \in \Lambda} \mathcal{L}_{\alpha}^{\lambda}\right)^{*}=\bigoplus_{\alpha \in \Pi, \lambda \in \Lambda} \mathcal{L}_{\alpha}^{\lambda}$, 4.2 gives us $I=I^{*}$.

Theorem 4.6. Let $\mathcal{L}$ be a perfect split involutive regular Hom-Lie color algebra of maximal length and root-multiplicative. Then $\mathcal{L}$ is simple if and only if it has all its nonzero roots connected.

Proof. The first implication is Theorem 3.6-(2). To prove the converse, consider $I$ a nonzero ideal of $\mathcal{L}=\mathcal{H} \oplus\left(\bigoplus_{\alpha \in \Pi} \mathcal{L}_{\alpha}\right)$. By $(4.7)$, we have $\mathcal{H} \subset I$. Given any $\alpha \in \Pi$, by the fact that $\alpha \neq 0$ and the maximal length of $\mathcal{L}$ we have

$$
\left[\mathcal{H}^{0}, \mathcal{L}_{\alpha \phi^{-1}}\right]=\mathcal{L}_{\alpha} \subset I
$$

and so $\bigoplus_{\alpha \in \Pi} \mathcal{L}_{\alpha} \subset I$. From here and $\mathcal{H} \subset I$, we conclude that $I=\mathcal{L}$. Therefore, $\mathcal{L}$ is simple.

Theorem 4.7. Let $\mathcal{L}$ be a perfect split involutive regular Hom-Lie color algebra of maximal length and root-multiplicative. Then

$$
\mathcal{L}=\bigoplus_{[\alpha] \in \Pi / \sim} I_{[\alpha]}
$$

where any $I_{[\alpha]}$ is a minimal involutive ideal of $\mathcal{L}$, and each one being a simple split involutive regular Hom-Lie color algebra having all its nonzero roots connected. 
Proof. By Corollary 3.9. we can write $\mathcal{L}=\bigoplus_{[\alpha] \in \Pi / \sim} I_{[\alpha]}$ as direct sum of the family of ideals

$$
I_{[\alpha]}=I_{0,[\alpha]} \oplus \mathcal{V}_{[\alpha]}=\sum_{\beta \in[\alpha]}\left[\mathcal{L}_{\beta},\left(\mathcal{L}_{\beta}\right)^{*}\right] \oplus\left(\bigoplus_{\beta \in[\alpha]} \mathcal{L}_{\beta}\right),
$$

where each $I_{[\alpha]}$ is a split involutive regular Hom-Lie color algebra having as root system $\Pi_{I_{[\alpha]}}=[\alpha]$. In order to apply Theorem 4.6 to each $I_{[\alpha]}$, we have to observe that the root-multiplicativity of $\mathcal{L}$ and Lemma 3.5 show that $\Pi_{I_{[\alpha]}}$ has all of its elements $\Pi_{I_{[\alpha]}}$-connected, that is, connected through connections contained in $\Pi_{I_{[\alpha]}}$. We also get that any of the $I_{[\alpha]}$ is root-multiplicative as consequence of the rootmultiplicativity of $\mathcal{L}$. Clearly, $I_{[\alpha]}$ is of maximal length, and finally $Z_{I_{[\alpha]}}\left(I_{[\alpha]}\right)=0$, as consequence of Lemma 3.5, Theorem 4.6 , and $Z(\mathcal{L})=0$. We can therefore apply Theorem 4.6 to any $I_{[\alpha]}$ so as to conclude that $I_{[\alpha]}$ is simple. It is clear that the decomposition $\mathcal{L}=\bigoplus_{[\alpha] \in \Pi / \sim} I_{[\alpha]}$ satisfies the assertions of the theorem.

\section{EXAMPLE}

In this section, an example is provided to clarify the results in Sections 3 and 4. The process of the example is described in four steps as follows.

Step 1 (Lie color algebra pso $(2 m+1,2 n)$ ). Let $\Lambda=\mathbb{Z}_{2} \times \mathbb{Z}_{2}$ and the skewsymmetric bi-character on $\Lambda$ is defined as

$$
\epsilon: \Lambda \times \Lambda \longrightarrow \mathbb{K} \backslash\{0\} ; \quad \epsilon(a, b)=(-1)^{a . b},
$$

where $a=\left(a_{1}, a_{2}\right)$ and $b=\left(b_{1}, b_{2}\right)$ are elements of $\mathbb{Z}_{2} \times \mathbb{Z}_{2}$ such that

$$
a+b=\left(a_{1}+b_{1}, a_{2}+b_{2}\right), \quad a \cdot b=a_{1} b_{1}+a_{2} b_{2} .
$$

As a linear space, the $\Lambda$-graded Lie superalgebra $\mathcal{L}$ is a direct sum of four graded components:

$$
\mathcal{L}=\bigoplus_{\lambda \in \Lambda} \mathcal{L}^{\lambda}=\mathcal{L}^{(0,0)} \oplus \mathcal{L}^{(0,1)} \oplus \mathcal{L}^{(1,0)} \oplus \mathcal{L}^{(1,1)}
$$

If $\mathcal{L}$ admits a $\epsilon$-bracket denoted by [.,.], satisfying the identities $\Lambda$-grading, $\epsilon$-symmetry and $\epsilon$-Jacobi, then $\mathcal{L}$ is referred to as a $\mathbb{Z}_{2} \times \mathbb{Z}_{2}$-graded Lie superalgebra (for more details see [13]). By the $\epsilon$-symmetry identity, the $\epsilon$-bracket for homogeneous elements is either a commutator or an anticommutator which we will denote by $[.,$.$] and \{.,$.$\} , respectively. Note that \mathcal{L}^{(0,0)}$ is a Lie superalgebra and that $\mathcal{L}^{(0,0)} \oplus \mathcal{L}^{(0,1)}$ and $\mathcal{L}^{(0,0)} \oplus \mathcal{L}^{(1,0)}$ are Lie-subsuperalgebras of the $\mathbb{Z}_{2} \times \mathbb{Z}_{2^{-}}$ graded Lie superalgebra $\mathcal{L}$. However, we will say that $\mathcal{L}$ is a Lie color algebra. Let us now construct the $\mathbb{Z}_{2} \times \mathbb{Z}_{2}$-graded Lie color algebra $\mathcal{L}=p s o(2 m+1,2 n)$. Its matrix form is closely related to, but diffrent from, that of the orthosymplectic Lie superalgebra $\operatorname{osp}(2 m+1,2 n)$. The Lie color algebra $\mathcal{L}=p s o(2 m+1,2 n)$ can be 
defined as the set of all block matrices of the form

$$
\left(\begin{array}{cc|c|cc}
a & b & u & x & y \\
c & a^{t} & v & z & w \\
\hline-v^{t} & -u^{t} & 0 & r & s \\
\hline-w^{t} & -y^{t} & s^{t} & d & e \\
z^{t} & x^{t} & -r^{t} & f & -d^{t}
\end{array}\right),
$$

where $a$ is any $(m \times n)$-matrix, $b$ and $c$ are skew-symmetric $(m \times m)$-matrices, $u$ and $v$ are $(m \times 1)$-matrices, $x, y, z, w$ are $m \times n$-matrices, $r$ and $s$ are $(1 \times n)$-matrices, $d$ is any $(n \times n)$-matrix and finally, $e$ and $f$ are symmetric $(n \times n)$-matrices. The $\mathbb{Z}_{2} \times \mathbb{Z}_{2}$ grading for matrices of $\mathcal{L}$ of the form (5.1) is determined by

$$
\mathcal{L}:=\left(\begin{array}{c|c|c}
\mathcal{L}^{(0,0)} & \mathcal{L}^{(1,1)} & \mathcal{L}^{(0,1)} \\
\hline \mathcal{L}^{(1,1)} & 0 & \mathcal{L}^{(1,0)} \\
\hline \mathcal{L}^{(0,1)} & \mathcal{L}^{(1,0)} & \mathcal{L}^{(0,0)}
\end{array}\right) .
$$

Denote by $e_{i, j}$ the matrix with zero everywhere except a 1 on position $(i, j)$, where the row and the column indices run from 1 to $2 m+2 n+1$. Similar to the $o s p(2 m+1,2 n)$ algebra, we introduce the following elements:

$$
\begin{aligned}
& c_{j}^{+}=\sqrt{2}\left(e_{j, 2 m+1}-e_{2 m+1, m+j}\right), \\
& c_{j}^{-}=\sqrt{2}\left(e_{2 m+1, j}-e_{m+j, 2 m+1}\right),
\end{aligned}
$$

for $j=1,2,3, \ldots, m$, and

$$
\begin{aligned}
& c_{m+j}^{+}=\sqrt{2}\left(e_{2 m+1,2 m+1+n+j}+e_{2 m+1+j, 2 m+1}\right), \\
& c_{m+j}^{-}=\sqrt{2}\left(e_{2 m+1,2 m+1+j}-e_{2 m+1+n+j, 2 m+1}\right),
\end{aligned}
$$

for $j=1,2,3, \ldots, n$.

In [13], Tolstoy proved that the $\mathbb{Z}_{2} \times \mathbb{Z}_{2}$-graded Lie superalgebra $\mathcal{L}$ is defined by $2 m+2 n$ generators $c_{j}^{ \pm}(j=1,2,3, \ldots, m)$ and $c_{k+m}^{ \pm}(k=1,2,3, \ldots, n)$, subject to the relations

$$
\begin{aligned}
& {\left[\left[c_{j}^{\mu}, c_{k}^{\eta}\right], c_{l}^{\nu}\right]=|\nu-\eta| \delta_{k l} c_{j}^{\mu}-|\nu-\mu| \delta_{j l} c_{k}^{\eta},} \\
& {\left[c_{m+j}^{\mu}, c_{m+k}^{\eta}, c_{m+l}^{\nu}\right]=(\nu-\mu) \delta_{j l} c_{m+k}^{\eta}+(\nu-\eta) \delta_{k l} c_{m+j}^{\mu},} \\
& {\left[c_{j}^{\mu}, c_{m+k}^{\eta}\right], c_{m+l}^{\nu}=-|\nu-\mu| \delta_{j l} c_{m+k}^{\eta}, \quad\left[c_{j}^{\mu}, c_{m+k}^{\eta}, c_{m+l}^{\nu}\right]=(\nu-\eta) \delta_{k l} c_{j}^{\mu},}
\end{aligned}
$$

where $j, k \in\{1,2,3, \ldots, m\}$ and $\mu, \eta, \nu \in\{ \pm\}$ is isomorphic to $p s o(2 m+1,2 n)$.

Step 2 (Involutive Hom-Lie color algebra $\operatorname{osp}(2 m+1,2 n)$ ). By using Theorem 3.14 in [20], we construct an involutive Hom-Lie color algebra from the Lie color algebra $p s o(2 m+1,2 n)$. For this purpose, we consider an even automorphism $\phi: p s o(2 m+1,2 n) \longrightarrow p s o(2 m+1,2 n)$ such that $\phi^{2}=i d$. More precisely, $\phi$ is an involution given by

$$
\begin{aligned}
& \phi\left(e_{2 m+1+j, k}\right)=-e_{2 m+1+j, k}, \quad j=1,2,3, \ldots, 2 n \text { and } k=1,2,3, \ldots, 2 m, \\
& \phi=i d, \quad \text { otherwise. }
\end{aligned}
$$


Notice that the image of the algebra $p s o(2 m+1,2 n)$ under the even linear map $\phi$ is exactly $\mathcal{L}=\operatorname{osp}(2 m+1,2 n)$, which can be defined as a set of all block matrices of the form (5.1) and the $\mathbb{Z}_{2} \times \mathbb{Z}_{2}$ grading of $\mathcal{L}$ for matrices of the form $(5.2)$. One can easily check that the tuple $\left(\mathcal{L}=\operatorname{osp}(2 m+1,2 n),[., .]_{\phi}=\phi \circ[.,],. \phi, \epsilon\right)$ is an involutive Hom-Lie color algebra exactly related to the orthosymplectic Lie superalgebra $\operatorname{osp}(2 m+1,2 n)$.

Step 3 (Root space decomposition). As a basis in the maximal involutive abelian subalgebra $\mathcal{H}$ of $(\mathcal{L}, \phi)$, consider

$$
\begin{gathered}
h_{i}=e_{i, i}-e_{m+i, m+i}=\frac{1}{2}\left[c_{i}^{-}, c_{i}^{+}\right], \quad i=1,2,3, \ldots, m \\
h_{m+j, m+j}=e_{2 m+1+j, 2 m+1+j}-e_{2 m+1+n+j, 2 m+1+n+j} \\
=\frac{1}{2}\left[c_{m+j, m+j}^{-}, c_{m+j, m+j}^{+}\right], \quad j=1,2,3, \ldots, n,
\end{gathered}
$$

which belong to $\mathcal{L}_{0}^{(0,0)}$. They span the space $\mathcal{H}$ of diagonal matrices in $\mathcal{L}$. That is,

$$
\mathcal{H}=\operatorname{span}_{\mathbb{K}}\left\{h_{i}, h_{m+j}: i=1,2,3, \ldots, m ; j=1,2,3, \ldots, n\right\} .
$$

In terms of dual basis, let $\left\{\varepsilon_{i}, \delta_{j}: i=1,2,3, \ldots, m ; j=1,2,3, \ldots, n\right\}$ be the dual basis of $\mathcal{H}^{*}$ given by

$$
\varepsilon_{i}\left(h_{k}\right)=\delta_{i k}, \quad \varepsilon_{i}\left(h_{m+j}\right)=0 ; \quad \delta_{j}\left(\varepsilon_{i}\right)=0, \quad \delta_{j}\left(h_{i}\right)=\delta_{j i}, \quad \text { and } \varepsilon_{2 m+2 n+1}=0 .
$$

Then, the root vectors and the corresponding root spaces are given by:

$$
\begin{aligned}
& e_{j, k}-e_{m+k, m+j} \in \mathcal{L}_{\varepsilon_{j}-\varepsilon_{k}}^{(0,0)}, \quad j \neq k=1,2,3, \ldots, m \\
& e_{j, m+k}-e_{k, m+j} \in \mathcal{L}_{\varepsilon_{j}+\varepsilon_{k}}^{(0,0)}, j<k=1,2,3, \ldots, m \\
& e_{m+j, k}-e_{m+k, j} \in \mathcal{L}_{-\left(\varepsilon_{j}+\varepsilon_{k}\right)}^{(0,0)}, \quad j<k=1,2,3, \ldots, m \\
& e_{j, 2 m+1}-e_{2 m+1, m+j} \in \mathcal{L}_{\varepsilon_{j}}^{(1,1)}, \quad j=1,2,3, \ldots, m \\
& e_{m+j, 2 m+1}-e_{2 m+1, j} \in \mathcal{L}_{-\varepsilon_{j}}^{(1,1)}, \quad j=1,2,3, \ldots, m \\
& e_{2 m+1+j, 2 m+1+k}-e_{2 m+1+n+k, 2 m+1+n j j} \in \mathcal{L}_{\delta_{j}-\delta_{k}}^{(0,1)}, \quad j \neq k=1,2,3, \ldots, n \\
& e_{2 m+1+j, 2 m+1+n+k}+e_{2 m+1+k, 2 m+1+n+j} \in \mathcal{L}_{\delta_{j}+\delta_{k}}^{(0,0)}, \quad j \neq k=1,2,3, \ldots, n \\
& e_{2 m+1+n+j, 2 m+1+k}+e_{2 m+1+n+k, 2 m+1+j} \in \mathcal{L}_{-\left(\delta_{j}+\delta_{k}\right)}^{(0,0)}, \quad j \neq k=1,2,3, \ldots, n \\
& e_{j, 2 m+1+k}-e_{2 m+1+n+k, m+j} \in \mathcal{L}_{\varepsilon_{j}-\delta_{k}}^{(0,1)}, j=1,2, \ldots, m ; k=1,2,3, \ldots, n \\
& e_{m+j, 2 m+1+k}-e_{2 m+1+n+k, j} \in \mathcal{L}_{-\left(\varepsilon_{j}+\delta_{k}\right)}^{(0,1)}, \quad j=1,2, \ldots, m ; k=1,2,3, \ldots, n \\
& e_{2 m+1,2 m+1+k}-e_{2 m+1+n+k, 2 m+1} \in \mathcal{L}_{-\delta_{k}}^{(1,0)}, \quad k=1,2,3, \ldots, n \\
& e_{j, 2 m+1+n+k}+e_{2 m+1+k, m+j} \in \mathcal{L}_{\varepsilon_{j}+\delta_{k}}^{(0,1)}, \quad j=1,2, \ldots, m ; k=1,2,3, \ldots, n \\
& e_{m+j, 2 m+1+n+k}-e_{2 m+1+k, j} \in \mathcal{L}_{-\varepsilon_{j}+\delta_{k}}^{(0,1)}, \quad j=1,2, \ldots, m ; k=1,2,3, \ldots, n \\
& e_{2 m+1,2 m+1+n+k}-e_{2 m+1+k, 2 m+1} \in \mathcal{L}_{\delta_{k}}^{(1,0)}, \quad k=1,2,3, \ldots, n .
\end{aligned}
$$


The set of roots is given by

$\Pi=\left\{ \pm \varepsilon_{j}, \pm\left(\varepsilon_{j} \pm \varepsilon_{k}\right), \pm \delta_{k}, \pm\left(\delta_{j} \pm \delta_{k}\right), \pm\left(\varepsilon_{j} \pm \delta_{k}\right): j=1,2, \ldots, m ; k=1,2,3, \ldots, n\right\}$.

So, we have a split involutive Hom-Lie color algebra with respect to $\mathcal{H}$, given by

$$
\mathcal{L}=\mathcal{H} \oplus\left(\bigoplus_{\lambda \in \Lambda} \bigoplus_{\alpha \in \Pi} \mathcal{L}_{\alpha}^{\lambda}\right)
$$

Step 4 (Inner structure). By the root system of the split involutive Hom-Lie color algebra $\left(\mathcal{L}=\operatorname{osp}(2 m+1,2 n),[., .]_{\phi}, \phi, \epsilon\right)$ which is presented in $(5.3)$, we have just the case $k=1$ in Definition 3.1 which is equivalent to the fact that $\beta=\varepsilon \alpha \phi^{z}$, for some $z \in \mathbb{Z}$ and $\varepsilon \in\{ \pm 1\}$. Then, we have the equivalent classes on the set of nonzero roots by

$$
[\alpha]=\{\alpha,-\alpha\}, \quad \forall \alpha \in \Pi .
$$

Now, for any $\alpha \in \Pi$ we have

$$
\begin{aligned}
I_{0,[\alpha]}= & \sum_{\beta \in[\alpha], \lambda \in \Lambda}\left[\mathcal{L}_{\beta}^{\lambda}, \mathcal{L}_{-\beta}^{-\lambda}\right] \oplus \sum_{\beta \in[\alpha], \lambda, \mu \in \Lambda, \lambda+\mu \neq 0}\left[\mathcal{L}_{\beta}^{\lambda}, \mathcal{L}_{-\beta}^{\mu}\right] \\
& \oplus \sum_{j, k}\left[\mathcal{L}_{\varepsilon_{j}+\varepsilon_{k}}^{(0,0)}, \mathcal{L}_{-\left(\varepsilon_{j}+\varepsilon_{k}\right)}^{(0,0)}\right] \oplus \sum_{j}\left[\mathcal{L}_{\varepsilon_{j}}^{(1,1)}, \mathcal{L}_{-\varepsilon_{j}}^{(1,1)}\right] \\
& \oplus \sum_{j, k}\left[\mathcal{L}_{\delta_{j}+\delta_{k}}^{(0,0)}, \mathcal{L}_{-\left(\delta_{j}+\delta_{k}\right)}^{(0,0)}\right] \oplus \sum_{k}\left[\mathcal{L}_{\delta_{k}}^{(1,0)}, \mathcal{L}_{-\delta_{k}}^{(1,0)}\right] \\
& \oplus \sum_{j, k}\left[\mathcal{L}_{\varepsilon_{j}+\delta_{k}}^{(0,1)}, \mathcal{L}_{-\left(\varepsilon_{j}+\delta_{k}\right)}^{(0,1)}\right] \oplus \sum_{j, k}\left[\mathcal{L}_{\varepsilon_{j}-\delta_{k}}^{(0,1)}, \mathcal{L}_{-\left(\varepsilon_{j}-\delta_{k}\right)}^{(0,1)}\right],
\end{aligned}
$$

and

$$
\mathcal{V}_{[\alpha]}=\bigoplus_{\lambda \in \Lambda} \bigoplus_{\beta \in[\alpha]} \mathcal{L}_{\beta}^{\lambda}=\sum_{j, k} \mathcal{L}_{\varepsilon_{j}-\varepsilon_{k}}^{(0,0)} \oplus \sum_{j, k} \mathcal{L}_{\delta_{j}-\delta_{k}}^{(0,1)} .
$$

Finally, we have $I_{[\alpha]}$ the direct sum of the two graded subspaces above, that is,

$$
I_{[\alpha]}=I_{0,[\alpha]} \oplus \mathcal{V}_{[\alpha]} .
$$

This is the same involutive ideal of the split involutive Hom-Lie color algebra $\left(\mathcal{L}=\operatorname{osp}(2 m+1,2 n),[., .]_{\phi}, \phi, \epsilon\right)$ which credits to Theorem 3.6 Since $\mathcal{L}$ is perfect, we have

$$
\mathcal{L}=\bigoplus_{[\alpha] \in \Pi / \sim} I_{[\alpha]} .
$$

\section{REFERENCES}

[1] K. Abdaoui, F. Ammar, and A. Makhlouf, Constructions and cohomology of Hom-Lie color algebras, Comm. Algebra 43 (2015), no. 11, 4581-4612. MR 3422354.

[2] F. Ammar, I. Ayadi, S. Mabrouk, and A. Makhlouf, Quadratic color Hom-Lie algebras. Preprint, 2012. arXiv:1204.5155 [math.RA].

[3] M. J. Aragón Periñán and A. J. Calderón Martín, Split regular Hom-Lie algebras, J. Lie Theory 25 (2015), no. 3, 875-888. MR 3385000

[4] F. Ammar, Z. Ejbehi and A. Makhlouf, Cohomology and deformations of Hom-algebras, J. Lie Theory 21 (2011), no. 4, 813-836. MR 2917693. 
[5] J. Bergen and D. S. Passman, Delta ideals of Lie color algebras, J. Algebra 177 (1995), no. 3, 740-754. MR 1358484

[6] A. J. Calderón Martín, On split Lie algebras with symmetric root systems, Proc. Indian Acad. Sci. (Math. Sci.) 118 (2008), no. 3, 351-356. MR 2450239

[7] Y. Cao and L. Chen, On split regular Hom-Lie color algebras, Colloq. Math. 146 (2017), 143-155. MR 3570208.

[8] A. J. Calderón Martín, J. M. Sánchez Delgado, On the structure of split Lie color algebras, Linear Algebra Appl. 436 (2012), no. 2, 307-315. MR 2854872

[9] A. J. Calderón Martín and J. M. Sánchez Delgado, On the structure of split involutive Lie algebras, Rocky Mountain J. Math. 44 (2014), no. 5, 1445-1455. MR 3295637.

[10] J. Feldvoss, Representations of Lie colour algebras, Adv. Math. 157 (2001), no. 2, 95-137. MR 1813428

[11] R. Ree, Generalized Lie elements, Canad. J. Math 12 (1960), 439-502. MR 0114880

[12] V. Rittenberg and D. Wyler, Generalized superalgebras, Nuclear Phys. B 139 (1978), no. 3, 189-202. MR 0491859

[13] V. Rittenberg and D. Wyler, Sequences of $Z_{2} \oplus Z_{2}$ graded Lie algebras and superalgebras, J. Math. Phys. 19 (1978), no. 10, 2193-2200. MR 0507516.

[14] M. Scheunert, Generalized Lie algebras, J. Math. Phys. 20 (1979), no. 4, 712-720. MR 0529734

[15] M. Scheunert and R. B. Zhang, Cohomology of Lie superalgebras and their generalizations, J. Math. Phys. 39 (1998), no. 9, 5024-5061. MR 1643330

[16] M. Scheunert, The theoy of Lie superalgebras: an introduction, Lecture Notes in Mathematics, 716. Springer, Berlin, 1979. MR 0537441

[17] B. Sun, L. Chen, and Y. Liu, $T^{*}$-Extensions and abelian extensions Hom-Lie color algebras, Rev. Un. Mat. Argentina 59 (2018), no. 1, 123-142. MR 3825766.

[19] M. C. Wilson, Delta methods in enveloping algebras of Lie colour algebras, J. Algebra 175 (1995), no. 2, 661-696. MR 1339662.

[20] L. Yuan, Hom-Lie color algebra structures, Comm. Algebra 40 (2012), no. 2, 575-592. MR 2889483

[21] J. Zhang, C. Zhang, and Y. Cao, On the structure of split involutive regular Hom-Lie algebras, Oper. Matrices 11 (2017), no. 3, 783-792. MR 3655685

\section{Khalili}

Department of Mathematics, Faculty of Sciences, Arak University, Arak 385156-8-8349,

P.O. Box 879, Iran

V-Khalili@araku.ac.ir

Received: January 23, 2018

Accepted: September 11, 2018 